\title{
Extreme adaptive optics planet imager: XAOPI
}

Bruce A. Macintosh, James Graham, Lisa Poyneer, Gary Sommargren, Julia Wilhelmsen, et al.

Bruce A. Macintosh, James Graham, Lisa Poyneer, Gary Sommargren, Julia Wilhelmsen, Don Gavel, Steve Jones, Paul Kalas, James P. Lloyd, Russ Makidon, Scot Olivier, Dave Palmer, Jennifer Patience, Marshall Perrin, Scott Severson, Andrew Sheinis, Anand Sivaramakrishnan, Mitch Troy, J. Kent Wallace, "Extreme adaptive optics planet imager: XAOPI," Proc. SPIE 5170, Techniques and Instrumentation for Detection of Exoplanets, (19 November 2003); doi: 10.1117/12.506836

SPIE Event: Optical Science and Technology, SPIE's 48th Annual Meeting, 2003, San Diego, California, United States 


\title{
Extreme Adaptive Optics Planet Imager: XAOPI \\ Bruce Macintosh $^{* \text { ab }}$, James Graham ${ }^{\text {ac }}$, Lisa Poyneer ${ }^{\text {ab }}$, Gary Sommargren ${ }^{\text {ab }}$, Julia Wilhelmsen $^{\text {ab }}$, Don Gavel ${ }^{\text {ad }}$, Steve Jones ${ }^{\text {ab }}$, Paul Kalas ${ }^{\text {ac }}$, James Lloyd ${ }^{\text {af }}$, Russ Makidon ${ }^{\text {ag }}$, Scot Olivier ${ }^{\text {ab }}$, Dave Palmer ${ }^{\text {ab }}$, Jennifer Patience ${ }^{\text {af }}$, Marshall Perrin ${ }^{\text {ac }}$, Scott Severson ${ }^{\text {ad }}$, Andrew Sheinis ${ }^{\text {ad }}$, Anand Sivaramakrishnan ${ }^{\text {ag }}$, Mitchell Troy ${ }^{\text {ae }}$, Kent Wallace ${ }^{\text {ae }}$ \\ ${ }^{a}$ NSF Center for Adaptive Optics \\ ${ }^{b}$ Lawrence Livermore National Laboratory, 7000 East Ave., Livermore, CA 94551 \\ ${ }^{c}$ University of California, 601 Campbell Hall, Berkeley, CA 94720 \\ ${ }^{\mathrm{d}}$ University of California, 1156 High Street, Santa Cruz, CA 95064 \\ e Jet Propulsion Laboratory, 4800 Oak Grove Drive, Pasadena, CA 91109 \\ ${ }^{\mathrm{f}}$ California Institute of Technology, 210 East California Blvd, Pasadena, CA 91125 \\ ${ }^{\mathrm{g}}$ Space Telescope Science Institute, 3500 San Martin Drive, Baltimore, MD 21218
}

\begin{abstract}
Ground based adaptive optics is a potentially powerful technique for direct imaging detection of extrasolar planets. Turbulence in the Earth's atmosphere imposes some fundamental limits, but the large size of ground-based telescopes compared to spacecraft can work to mitigate this. We are carrying out a design study for a dedicated ultra-high-contrast system, the eXtreme Adaptive Optics Planet Imager (XAOPI), which could be deployed on an 8-10m telescope in 2007. With a 4096-actuator MEMS deformable mirror it should achieve Strehl $>0.9$ in the near-IR. Using an innovative spatially filtered wavefront sensor, the system will be optimized to control scattered light over a large radius and suppress artifacts caused by static errors. We predict that it will achieve contrast levels of $10^{7}-10^{8}$ at angular separations of 0.2-0.8" around a large sample of stars $(\mathrm{R}<7-10)$, sufficient to detect Jupiter-like planets through their near-IR emission over a wide range of ages and masses. We are constructing a high-contrast $\mathrm{AO}$ testbed to verify key concepts of our system, and present preliminary results here, showing an RMS wavefront error of $<1.3 \mathrm{~nm}$ with a flat mirror.
\end{abstract}

Keywords: Adaptive optics; extrasolar planets; MOEMS

\section{INTRODUCTION}

Ground-based adaptive optics (AO) direct imaging of planets was proposed by Angel ${ }^{1}$, who recognized that even in the presence of the large phase errors imposed by the Earth's atmosphere, an AO system operating on a 6-10 m telescope can achieve contrast levels sufficient for direct detection of Jovian planets. This field of ultra-high-contrast imaging is sometimes called "Extreme Adaptive Optics" (ExAO). Although more recent work $^{2,3}$ shows that some of the early assumptions were optimistic, it is still possible to design an AO system, optimized for high-contrast imaging rather than for general purposes, that can achieve contrasts of $10^{7}-10^{8}$ for a large sample of target stars $\left(\mathrm{m}_{R}<7\right)$, potentially detecting a large fraction of extrasolar planets. The NSF Center for Adaptive Optics has begun the design of such a system, the eXtreme Adaptive Optics Planet Imager (XAOPI). Figure 1 shows a simulated XAOPI image. The star is hidden behind a central occulting spot. XAOPI's spatially-filtered wavefront sensor allows it to remove mid-spatial-frequency wavefront aberrations introduced by the telescope and atmosphere without contamination due to aliasing effects (section 3.2). This produces a characteristic square null region, similar to predictions for spacebased imaging, but not normally seen even in simulations of ground-based AO with pupil-plane sensors.

\section{GROUND-BASED ADAPTIVE OPTICS HIGH-CONTRAST IMAGING}

Ground-based ExAO imaging has some similarities to space-based high-contrast coronagraphy, but also some key differences. Both require suppression of scattered light to allow detection of a faint planetary companion, but the relative importance of difference sources of scattered light vary. We will discuss in turn

\footnotetext{
*bmac@igpp.1lnl.gov
} 


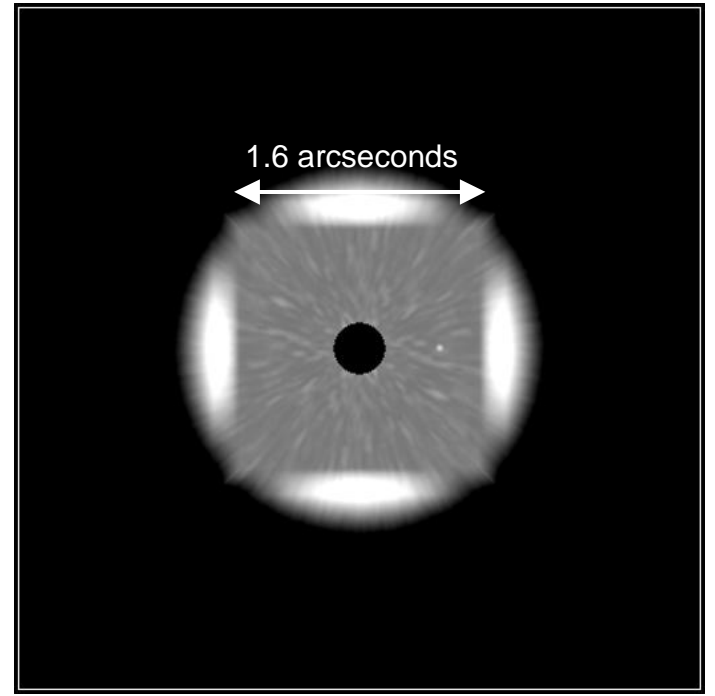

Figure 1: Simulated XAOPI image. This shows a simulated 15-minute H-band exposure of a solar-type star at a distance of $10 \mathrm{pc}$. A $8 \mathrm{M}_{\mathrm{J}}, 500 \mathrm{Myr}$ planet is located to the right of the star. each major source of scattered light or residual image noise in ExAO and compare these to conventional AO and/or space-based coronagraphy.

\subsection{Diffraction}

Light scattered by diffraction (due to the finite size of the telescope primary mirror) is a major issue in high-contrast space imaging, leading to a variety of coronagraph designs. This is particularly true since spacecraft primary mirrors are sharply constrained in size, to $<2 \mathrm{~m}$ for near-term missions intended to detect Jovian planets. Coronagraph design is particularly challenging for detection of objects at separations less than $\sim 4 \lambda / D$, where $\lambda$ is the imaging wavelength and $D$ the telescope diameter ${ }^{4,5}$. ExAO systems can operate on large ground-based 8-10m telescopes; as a result, a given angular scale corresponds to a much larger number of diffraction radii, and coronagraph design is simplified. This in turn can allow ExAO to operate at longer wavelengths such as the near-infrared (1-

$5 \mu \mathrm{m}$ ), where Jovian planets that are massive (>3 Jupiter masses) and/or young ( $<1$ Gyr) can be selfluminous ${ }^{6}$, with contrast levels of $10^{6}-10^{8}$ rather than $10^{9}$.

\subsection{Wavefront errors}

The greatest difference between ground-based AO and space coronagraphy, of course, are the large, rapidly-evolving phase errors (>1000 nm) imposed by the Earth's atmosphere. To achieve contrast levels of $10^{7}$ these need to be controlled to a few tens of $\mathrm{nm}$ over the relevant range of spatial frequencies, a reduction of a factor of 100. Wavefront control is therefore the critical requirement for ExAO. As in any high-contrast imaging, a wavefront error of a given spatial frequency $k$ (in cycles across the aperture) will scatter light primarily to a angular radius given by $k \lambda / D,{ }^{7}$ so that the key to detecting planets at moderate angular separations is control of mid-spatial frequency errors. In section 4.1 we will discuss the individual wavefront error sources for ExAO and the predicted values of these sources for XAOPI.

\subsection{Temporal evolution of wavefront errors}

Wavefront errors imposed by the Earth's atmosphere evolve rapidly with time; as a result, the wavefront measurement must be carried out and the correction applied in an extremely short time. For example, in the case of a single atmospheric turbulence layer with turbulence scale parameter (Fried parameter) $r_{0}$ moving with a wind velocity $v$, the residual error due to timelags for a $\mathrm{AO}$ system operating with a pure time delay $\tau_{\mathrm{s}}$ is $\sigma_{\mathrm{td}}^{2}=28.4\left(\tau_{\mathrm{s}} f_{g}\right)^{5 / 3}$ where $f_{g}$ is the Greenwood frequency $0.427 v / r_{0}$. As a result, ExAO systems must measure and correct the wavefront at rates $>1 \mathrm{kHz}$. This in turn imposes a requirement that the observed target be bright enough to provide a high-quality wavefront measurement in this short time, a requirement that becomes more difficult as the AO system subaperture size d becomes smaller. Classical AO systems, with $d \sim 60 \mathrm{~cm}$, can operate with wavefront references as dim as $\mathrm{m}_{\mathrm{R}}=12-14$ or with artificial reference beacons, but ExAO systems, with $d=10-30 \mathrm{~cm}$, require much brighter stars. This sets the ultimate limit on ExAO performance - while in principle an ExAO system could be constructed to achieve contrast levels of $10^{9}$ or higher, in practice such a system would operate only on stars brighter than $m_{R}=3-4$, severely limiting its science range ${ }^{2}$.

On the other hand, the rapid time evolution of wavefront errors does provide an advantage. The instantaneous point spread function with residual phase errors contains a halo broken up into individual speckles; as a result, companions can only be detected if their brightness is comparable to the brightness of the halo. Over long time periods the average wavefront error (with piston removed) approaches zero, which in turn causes the halo to become smoother, allowing detection of planets as small deviations from a smooth halo. A key question in the ultimate sensitivity of ExAO is the timescale on which this speckle 
decorrelation takes place. Angel ${ }^{8}$ suggested that the speckle pattern would be completely independent each update of the AO system, i.e. on timescales less than a millisecond, but Sivaramakrishnan et al. showed that in fact speckles due to each wavefront error source will evolve at their own independent rates ${ }^{3}$. As a result, the most critical wavefront errors are those that evolve slowly (such as atmospheric errors) while those that evolve rapidly (such as errors due to photon noise in the wavefront measurement) have a reduced effect on final sensitivity. Most critical of all are static or slowly evolving wavefront errors due to miscalibration of the AO system or unsensed optical errors, which can imprint a residual speckle pattern onto the halo that will not smooth with integration time. The requirement for these quasi-static errors in ExAO is in fact comparable, for a given contrast level, to the wavefront requirements for space coronagraphy.

\subsection{Wavefront sensing approaches}

Ground-based AO traditionally uses wavefront sensors (WFS) that operate in a plane conjugate to the system pupil. This has several advantages both conceptually, in that the sensor is conjugate to the phase corrector, and practically, in that a wavefront measurement in the pupil plane is wavelength-independent and hence can be easily carried out in broadband light. Most suggested spacecraft coronagraphic systems, by contrast, are intending to carry out wavefront measurements directly in the focal plane ${ }^{9}$.

Pupil-plane wavefront measurements have two difficulties that must be overcome in an ExAO system. The first is that finite sampling of the wavefront in the pupil plane can impose aliasing; uncorrectable high spatial frequency errors will be mis-measured as low/mid-frequency errors and applied as an erroneous correction. These additional mid-frequency errors cause AO systems to produce point-spread functions (PSFs) with a flat scattered light halo rather than a dark null region. XAOPI will use a spatially-filtered wavefront sensor (section 3.2) to overcome this otherwise-dominant source of scattered light. Secondly, since the wavefront sensor and the science camera are separate, the science image may be corrupted by unsensed optical errors in the non-common-path (NCP). These NCP errors can in principle be measured and removed by adjusting the wavefront control to a mean phase on the wavefront sensor that is the conjugate of the NCP errors, so that the science camera sees a flat wavefront while the WFS does not. Such a technique will work only if the non-common path errors are stable and the wavefront sensor provides an absolute measurement of the deviation from a flat wavefront, which is not always the case. As a result, NCP errors must be carefully controlled in an ExAO system.

\section{XAOPI CONCEPTUAL DESIGN}

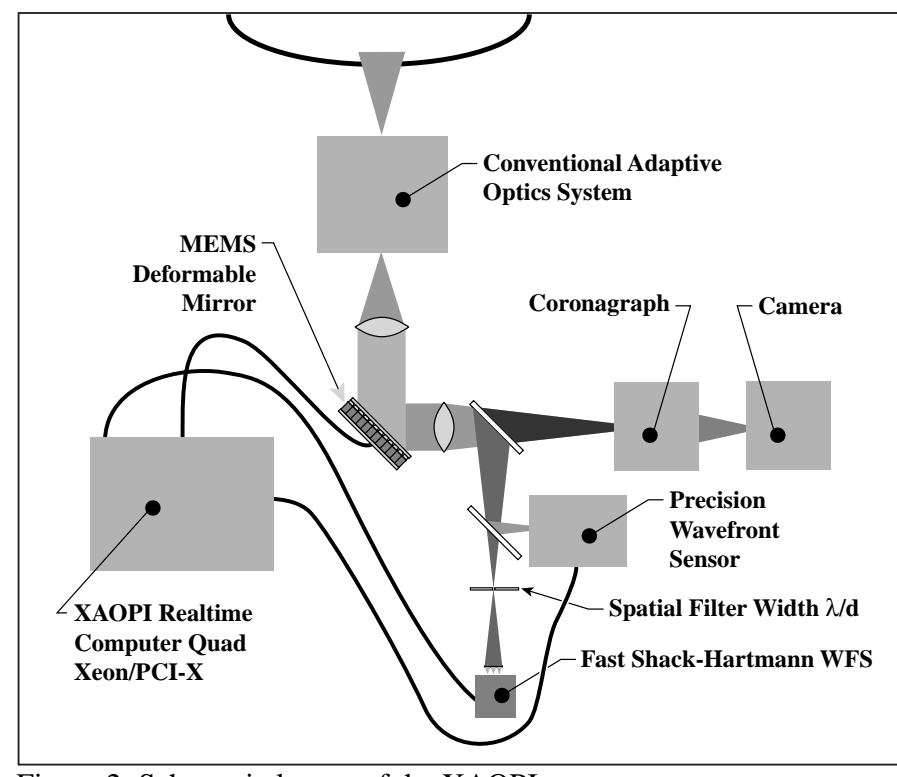

Figure 2: Schematic layout of the XAOPI system
XAOPI, the eXtreme Adaptive Optics Planet Imager, is a proposed planetdetecting 4096-actuator ExAO system being designed by the NSF Center for Adaptive Optics. We are currently carrying out a conceptual design study for this system, which could be deployed in 2007. XAOPI is being designed for use with the $10-\mathrm{m}$ W.M. Keck II telescope; we believe that our architecture, in particular the spatially filtered wavefront sensor (section 3.2), allows us to overcome quasi-static wavefront discontinuities due to the segmented Keck primary mirror. Figure 2 shows a schematic of the XAOPI layout. We will discuss each key component in turn, and in section 4 discuss the predicted performance of the system.

The XAOPI design has been driven 
by a careful analysis of the science requirements, analytic calculations of scattered light sources, simulations, and extrapolations of current technologies. The $\mathrm{d}=18 \mathrm{~cm}$ subaperture size, for example, is both a good match to expected MEMS technology and a good scientific compromise between smaller subapertures which would require very bright stars for full performance (e.g. the d 10 cm USAF AEOS system $^{10}$ ) and larger subapertures which would provide a marginal performance gain. We estimate that XAOPI will achieve full performance on target stars brighter than $m_{R}=7$ with good performance down to $\mathrm{m}_{\mathrm{R}}=10$.

\subsection{MEMS deformable mirror}

XAOPI requires a deformable mirror (DM) with $\sim 4000$ actuators. Conventional piezoelectric deformable mirrors have actuators $\sim 1 \mathrm{~cm}$ in size and cost $\sim \$ 1000 /$ actuator, prohibitively large and expensive for our purposes. Instead, we will use a micro-electro-mechanical-system (MEMS) deformable mirror. Micromachined out of silicon, such deformable mirrors are extremely compact (actuator spacing of $~ 300$ microns) and potentially inexpensive, with current costs of $\sim \$ 100-300$ per actuator driven primarily by external drive electronics. 1024-actuator continuous-facesheet MEMS have been demonstrated, e.g. by Boston Micromachines ${ }^{11}$. 4096-actuator MEMS can be constructed by scaling current designs; past that point, new designs with integrated electronics would be required. MEMS mirrors do, however, currently have limited stroke ( $\sim 1$ micron, with $\sim 4$ micron devices being tested). This is insufficient to fully correct atmospheric turbulence. As a result, a second deformable mirror may be needed to remove the large excursions in the wavefront. Our plan is to use the existing AO system at the observatory to serve this "woofer" function, while XAOPI provides the fine phase corrections. We have studied the interactions of the two AO systems and believe they can operate independently provided the coarse AO system operates at a much slower update rate ${ }^{12}$.

\subsection{Fast spatially-filtered wavefront sensor}

XAOPI's primary (fast) wavefront sensor must operate at $\sim 2.5 \mathrm{kHz}$ to achieve our performance goals. We intend to use Shack-Hartmann (S-H) wavefront sensor incorporating a spatial filter. Shack-Hartmann sensors have three significant limitations, which XAOPI will overcome. The first is computational; reconstructing a wavefront from S-H slope measurements with normal algorithms is computationally intensive (see section 3.5). The second is accuracy: $\mathrm{S}-\mathrm{H}$ wavefront sensors, particularly those operating with few pixels per subaperture, have a response that depends on the size of the spot as seen by the wavefront sensor. We will mitigate the effects of this through careful control of NCP errors, so the sensor is always operating close to null, and possibly with a second high-accuracy wavefront sensor (3.3.) The final limitation of current sensors is aliasing (section 2.4). We will sharply reduce aliasing through a novel spatially-filtered wavefront sensor (SFWFS.) Briefly, since high spatial frequencies correspond to wide angles in the PSF, we can remove these components by passing the wavefront sensor light through a square spatial filter whose width is set to pass only those spatial frequencies that are Nyquist sampled by the subapertures of the WFS, i.e. a filter of width $\lambda / d$ where $d$ is the subaperture size. This allows the wavefront sensor to see phase errors that have been low-pass filtered, which can be correctly measured and reconstructed with no aliasing. Detailed simulations ${ }^{13}$ show that this reduces scattered light within the controlled region by a factor of 50 or more, and can even correct for phase errors caused by phase discontinuities such as the segmented edges of the Keck primary mirror.

Our baseline S-H wavefront sensor will use a $128 \times 128$ pixel CCD with only $2 \times 2$ pixels per subaperture; no larger CCD that meets the readnoise and framerate requirements is currently available, though a $4 \times 4$ pixel subaperture system with an optimal centroider would reduce the sensitivity of the S-H to seeing changes.

\subsection{Slow high-accuracy wavefront sensor}

Since residual non-common-path errors and static errors can limit ExAO sensitivity (section 2.3), a requirement for ExAO is that the static wavefront errors, especially at mid spatial frequencies, be very small (see Table 1.) XAOPI will take several parallel approaches to controlling these. First, the optical design has very small non-common-path errors, and all the components in the non-common-path will be constructed with very high wavefront error requirements. Note that since optics after the focal-plane stop of the coronagraph do not contribute significantly to scattered light, the only optics to which this specification 
applies are the beamsplitter(s) and the wavefront sensor optics, in particular the lenslets. Construction of a beamsplitter that meets these requirements may be very challenging, however. Second, we will use phase retrieval or phase-contrast techniques to calibrate the internal NCP errors. A final concern is that the absolute gain of a Shack-Hartmann sensor operating with $2 \times 2$ pixels per subaperture depends on the size of the individual spots, which in turn depends on the atmospheric seeing. Although this is a weak effect since we have subaperture size $d<r_{0}$ at the wavefront sensing wavelength and hence our wavefront sensor spots will be nearly diffraction limited, it can still result in a fraction of the NCP errors leaking through as $r_{0}$ changes during observations. We will explore approaches to adjust the wavefront sensor gain dynamically to compensate for this. We will also explore incorporating an auxiliary wavefront sensor that provides an absolute wavefront measurement, such as a Mach-Zehnder interferometer ${ }^{8}$; operating at low update rates $(\sim 1 \mathrm{~Hz})$ with a small fraction of the available light, this sensor would provide a time-averaged absolute wavefront measurement that could be used to update the S-H sensor control point.

\subsection{Coronagraph and science camera}

As discussed in section 2, coronagraph requirements for XAOPI are much less demanding than for most proposed space-based planet finders, requiring only contrasts of $10^{7}-10^{8}$ at radii of $>5 \lambda / \mathrm{D}$. A classic Lyot coronagraph can achieve these levels with moderate throughput. We are exploring more sophisticated coronagraphs, especially hybrids combining focal-plane masking with apodization of the pupil. The coronagraph will be a self-contained warm unit to allow a variety of science instruments. The initial science camera will be a simple imager or a dual-channel instrument ${ }^{14}$, with a integral field unit as a possible upgrade path. The sensitivity predictions shown in figure 1 and 4 are for single-channel broad-band imaging and do not assume any speckle suppression due to dual-channel or multiwavelength imaging; such an observational mode could significantly increase contrast for bright targets, assuming extrasolar planets have strong spectral features similar to known T-dwarfs. Multichannel polarimetery ${ }^{15}$ would similarly increase sensitivity to polarized scattering from extrasolar debris disks.

\subsection{Realtime controller}

Classic vector-matrix-multiply (VMM) wavefront reconstruction algorithms scale computationally as the number of subapertures $N$ squared. For a system with several thousand subapertures this is strains the capabilities of current or near-future realtime computers. Instead, XAOPI will use a Fourier-based wavefront reconstruction algorithm ${ }^{16}$ that scales as $N \log N$. Figure 3 compares the computational requirements for the two approaches. A commercial system based around four Pentium XEON processors and PCI-X bus appears to satisfy our requirements.

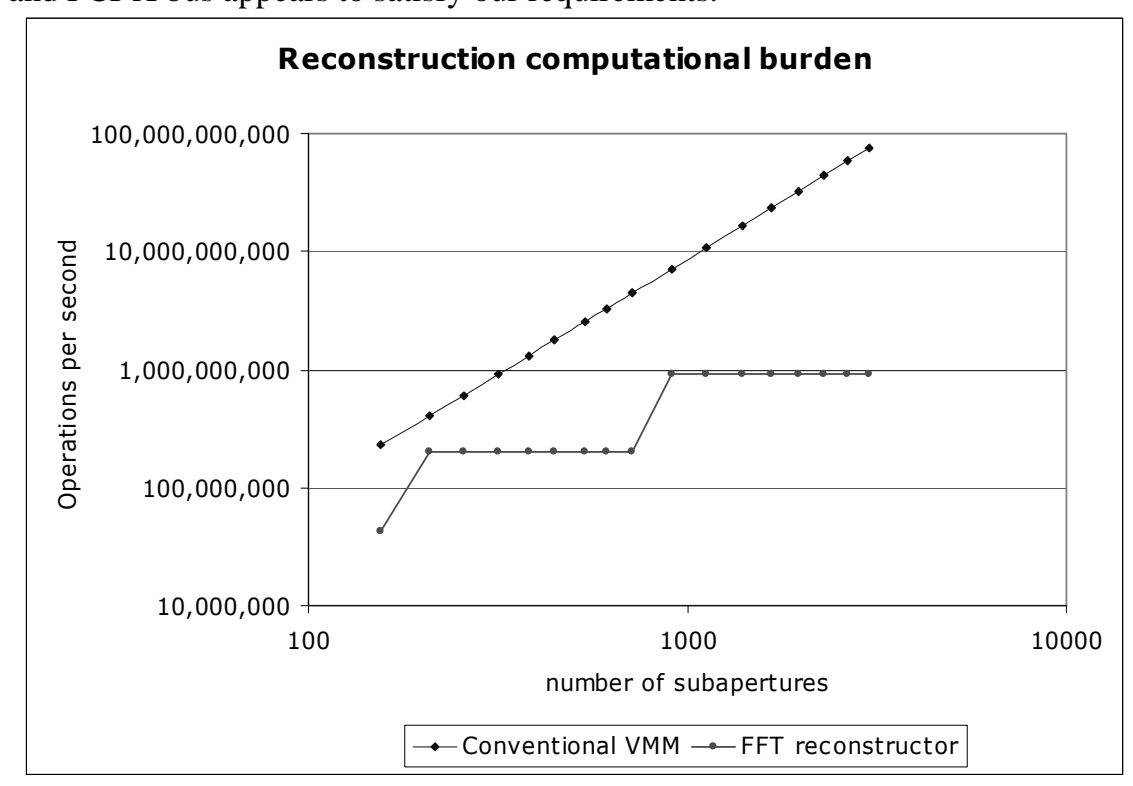

Figure 3: Computational requirements for VMM and FFT reconstructors. 


\section{XAOPI ERROR BUDGET AND PERFORMANCE}

Table 1 gives the XAOPI error budget, broken down as a function of wavefront spatial frequency $\mathrm{k}$ in cycles across the pupil. The crucial error terms are those in the mid-frequency range, which scatter light into the "dark hole" region. The table also gives the lifetime of speckles produced by each error source; the most crucial are those with long lifetimes, such as atmospheric errors, and the quasi-static errors.

\begin{tabular}{lrrrr} 
Error & $\begin{array}{l}\text { Low-freq } \\
k<5\end{array}$ & $\begin{array}{l}\text { Mid-freq } \\
5<k<32\end{array}$ & $\begin{array}{l}\text { High-freq } \\
k>32\end{array}$ & Speckle lifetime \\
\hline Atmosphere fitting and aliasing & & $2 \mathrm{~nm}$ & $44 \mathrm{~nm}$ & $\sim 0.4 \mathrm{~s}$ \\
Telescope primary mirror & & $1 \mathrm{~nm}$ & $22 \mathrm{~nm}$ & Minutes \\
Telescope vibration & $5 \mathrm{~nm}$ & $3 \mathrm{~nm}$ & $16 \mathrm{~nm}$ & $\sim 0.03 \mathrm{~s}$ \\
Internal calibration & $20 \mathrm{~nm}$ & $21 \mathrm{~nm}$ & $14 \mathrm{~nm}$ & $\begin{array}{r}\text { Static } \\
\text { Atmospheric bandwidth }\end{array}$ \\
WFS measurement & & $30 \mathrm{~nm}$ & & $\sim 0.005 \mathrm{~s}$ \\
DM & $3 \mathrm{~nm}$ & $2 \mathrm{~nm}$ & $30 \mathrm{~nm}$ & $\begin{array}{r}\text { Static } \\
\text { Changes in NCP errors }\end{array}$ \\
Total & $\mathbf{2 1 ~ n m}$ & $\mathbf{3 7 ~ n m}$ & $\mathbf{5 9} \mathbf{~ n m}$ & Minutes-hours \\
Overall Total & & $\mathbf{7 3 ~ n m}$ & & \\
Strehl Ratio @ 1.65 $\mu \mathrm{mm}$ & & $\mathbf{0 . 9 3}$ & &
\end{tabular}

Table 1: XAOPI error budget, divided into different categories as a function of spatial frequency $\mathrm{k}$ in cycles/pupil. This assumes a $\mathrm{m}_{\mathrm{R}}=7$ target star, an update rate of $2500 \mathrm{kHz}$, and 62 subapertures across the W.M. Keck I telescope.

\subsection{Wavefront error sources}

Atmospheric fitting and aliasing: Classic atmospheric fitting error in AO refers to the high-frequency components of the atmospheric phase that cannot be measured by the wavefront sensor or corrected by the deformable mirror. Rigaut et al. ${ }^{17}$ recognized that there is an additional error term due to aliasing of highspatial frequency components beyond the Nyquist frequency of the wavefront sensor. This error is approximately $1 / 3$ of the fitting error, but is the dominant source of mid-frequency errors in a classic AO system. XAOPI's spatially-filtered wavefront sensor will reject almost all of this error; see Poyneer and Macintosh ${ }^{13}$ for detailed simulations.

Telescope primary mirror and vibration: The segmented Keck primary mirror imposes additional error sources due to low-frequency aberrations of the segments and segment piston/tip/tilt errors, both static errors and $\sim 30 \mathrm{~Hz}$ segment vibrations. Again, as discussed in Poyneer and Macintosh ${ }^{13}$, the SFWFS allows us to measure and correct the mid-frequency components of even phase discontinuities.

Internal calibration: The $\mathrm{AO}$ system must be calibrated to remove non-common-path optical errors.

Atmospheric bandwidth: See section 2.3

WFS measurement: This represents the error due to finite signal-to-noise in the wavefront sensor. Although it is a significant source of mid-frequency error, it produces a rapidly-evolving speckle pattern (changing at approximately $1 / 10$ of the system update rate for a closed-loop controller.)

$D M$ : This represents the uncorrectable high-frequency errors in the deformable mirror.

Changes in NCP errors: This represents the time evolution in the correction of non-common-path errors, due to temperature changes in the optics and changes in the response of the wavefront sensor due to atmospheric seeing changes.

\subsection{Science reach}

The true metric for the scientific utility of a system like XAOPI is its ability to answer key scientific questions, such as the population of extrasolar planets in the wide (a>5 AU) orbits that are difficult to probe with indirect techniques. For our system the detection reach is complex to evaluate, since the detectability of a planet depends on its age and mass (which determine near-infrared flux), angular separation from its primary star, and the magnitude and distance of the parent star. We are using Monte Carlo simulations to evaluate XAOPI's ability to detect simulated planet populations. These will be discussed in detail in an upcoming paper $^{18}$; briefly, we take simulated stellar populations for the solar neighborhood based on conservative assumptions about the local star formation history, and planet populations generated from 
maximum-likelihood extrapolations of the known radial velocity planets. These in turn generate a simulated planet population (expressed as contrast vs. radius) which can be compared to simulations of XAOPI performance. Figure 4 shows one such simulation; a magnitude-limited XAOPI survey of the solar neighborhood would discover extrasolar planets around $7.5 \%$ of the target stars in one-hour integrations.

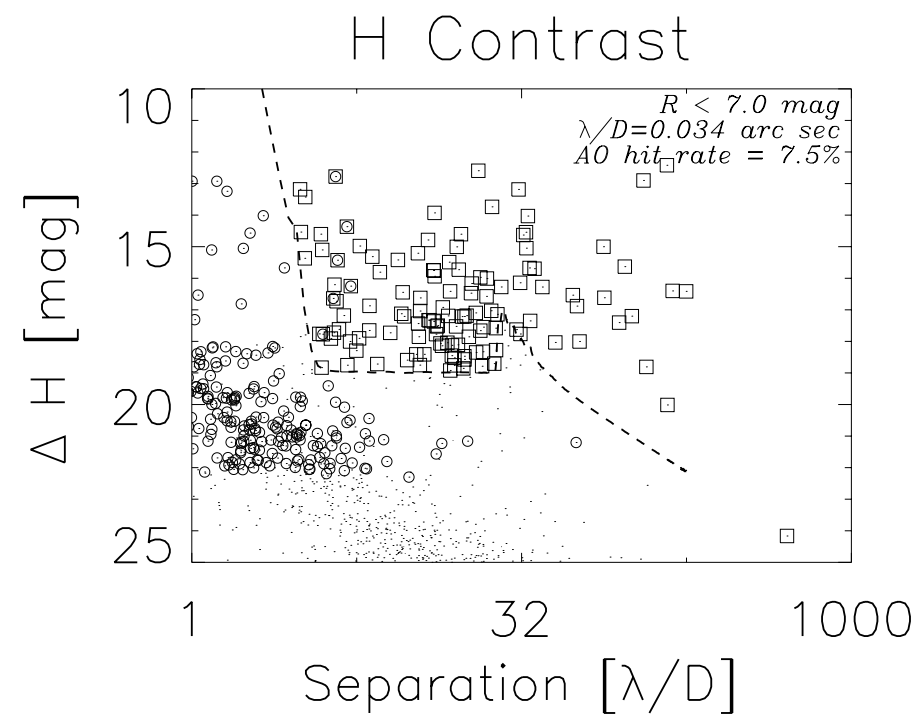

Figure 4: Monte Carlo simulation of XAOPI observations of the solar neighborhood. Each dot represents a planet. Circled dots would be detected by radial-velocity surveys. The dashed line shows the 5-sigma contrast predicted for XAOPI in a one-hour integration. Planets above this line (marked by squares) would be found by XAOPI.

Since planets are most detectable when young, it makes sense to preferentially target young stars. A significant excess of such stars are known to exist in the solar neighborhood ${ }^{19}$. By targeting a young association such as the TW Hydra or Beta Pictoris groups, up to $60 \%$ of the simulated planet population can be detected.

A second science goal is direct detection of circumstellar debris disks. Current direct imaging detections of debris disks are limited to cases with very high dust masses, such as HR $4796^{20}$ and/or favorable geometries such as Beta Pictoris. Within the null region (<0.8”) XAOPI will be able to image debris disks with surface brightnesses a factor of 100 lower than HR4796.

\section{EXAO TESTBED}

Since XAOPI's wavefront control requirements, particularly the static internal and calibration requirements, are an order of magnitude more exacting than any AO system to date, we are constructing a laboratory testbed to verify our ability to reach these levels of accuracy. This testbed is located at UC Santa Cruz in the new Laboratory for Adaptive Optics, funded by a donation from the Gordon and Betty Moore foundation.

Our approach to the testbed is to begin with an optically extremely simple system with extremely high quality metrology and to add complexity and functionality as each stage meets its performance requirements. In its initial configuration (Figure 5), the testbed optics consist of a single high-quality lens and a superpolished flat mirror. A simulated source is injected via fiber 1 and imaged on a CCD. Alternatively, the imaging CCD can be moved out and a second fiber embedded in a superpolished reflecting surface can be inserted. This second fiber is coherent with the first and acts as a Phase-Shifting Diffraction Interferometer (PSDI) ${ }^{21,22}$. This instrument provides sub-nm absolute wavefront measurements of an optical system with only the insertion of fibers into the input and output focal planes. Briefly, light 
from the first fiber passes through the optical system interferes with the spherical reference wave from the second fiber to form an interferogram on a CCD located some distance after the focus. The interferogram provides a measurement of the phase and amplitude in this plane, which is then numerically propagated to the surface of interest. Since no reference or additional optics are involved, this provides an absolute measurement of the phase, and has demonstrated accuracy better than $0.3 \mathrm{~nm}$ in characterizing optics for extreme ultra-violet lithography. The PSDI will form the cornerstone of our testbed, providing absolute wavefront measurements throughout the development of the system.

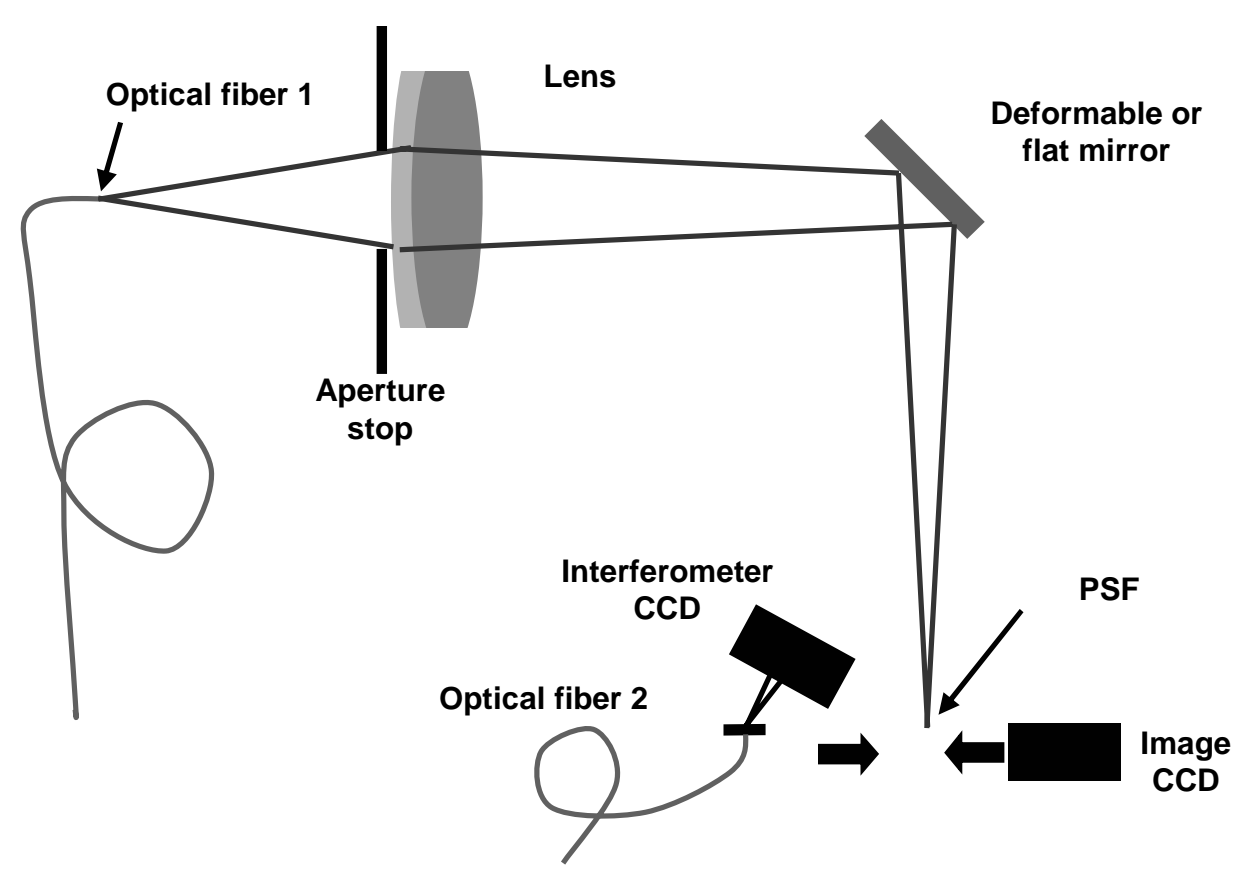

Figure 5: Schematic layout of the first-generation LAO testbed. The system can be operated in PSF measurement mode, with an imaging CCD in the focal plane, or in wavefront measurement mode, with a second fiber, coherent with the first, forming phase-shifting diffraction interferometer (PSDI).

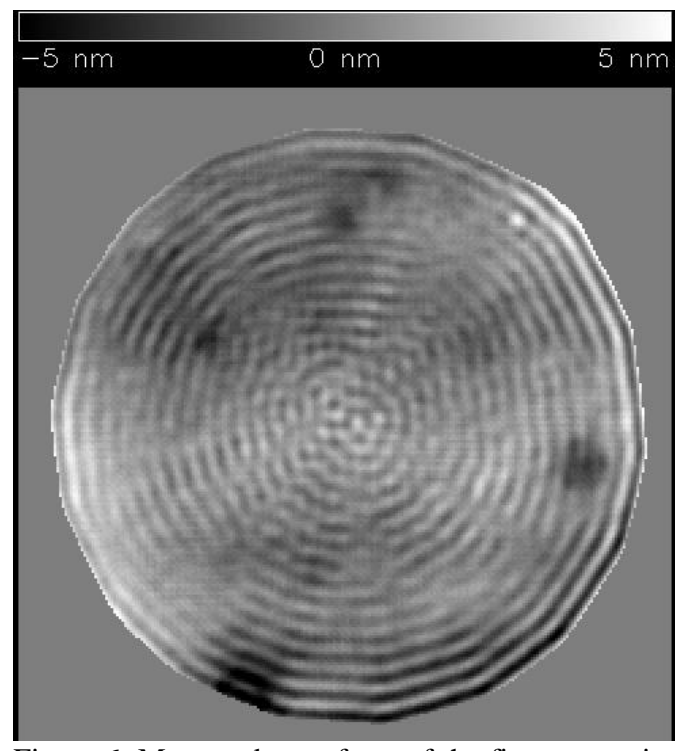

Figure 6: Measured wavefront of the first-generation testbed. The RMS wavefront error is $<1.3 \mathrm{~nm}$.
In its current configuration we measure a RMS wavefront error of $<1.3 \mathrm{~nm}$, with $0.7 \mathrm{~nm}$ of astigmatism and $0.8 \mathrm{~nm}$ of mid-frequency errors. The measurement contains $\sim 0.5 \mathrm{~nm}$ of spurious ringing due to small focus errors and low signal to noise at the edges of the CCD used for wavefront measurement. Figure 6 shows the measured wavefront.

Measurements of the system PSF were made with a 12-bit camera, which limits the dynamic range of a single image. The intensity of the laser was modified with a series of ND filters to build up a PSF measurement with dynamic range $\sim 10^{7}$, the ultimate sensitivity being limited by dark current and bias fluctuations in the uncooled camera. Since the optical layout has no coronagraph and an unapodized pupil, the PSF is dominated by the Airy pattern. Nonetheless this serves to set a lower limit on the contrast that would be achieved with a coronagraphic or apodized system; the PSF in the regions where the diffraction pattern goes to zero is set by wavefront 
errors in what is described as the "halo" term in the Taylor expansion of the PSF, the fundamental limit to contrast regardless of how diffraction is suppressed with a coronagraph or apodization ${ }^{7,3}$; strong phase errors fill in the Airy nulls. In our experiment, finite sampling prevents us from seeing the full depth of the PSF in the Airy nulls, so we can only produce an upper limit on the contrast. Figure 7 shows a slice through the PSF. Figure 8 shows a slice through a simulated PSF generated with the measured wavefront and a PSF generated with the same aperture and no wavefront errors; the two curves are nearly identical, showing that finite sampling of the diffraction rather than wavefront errors limit contrast. Calculations with the measured phase error show that if diffraction was suppressed, contrast would reach $10^{8}$. The testbed therefore provides an ideal arrangement, even with a flat mirror, for testing pupil apodization concepts, providing their implementation does not introduce wavefront errors.

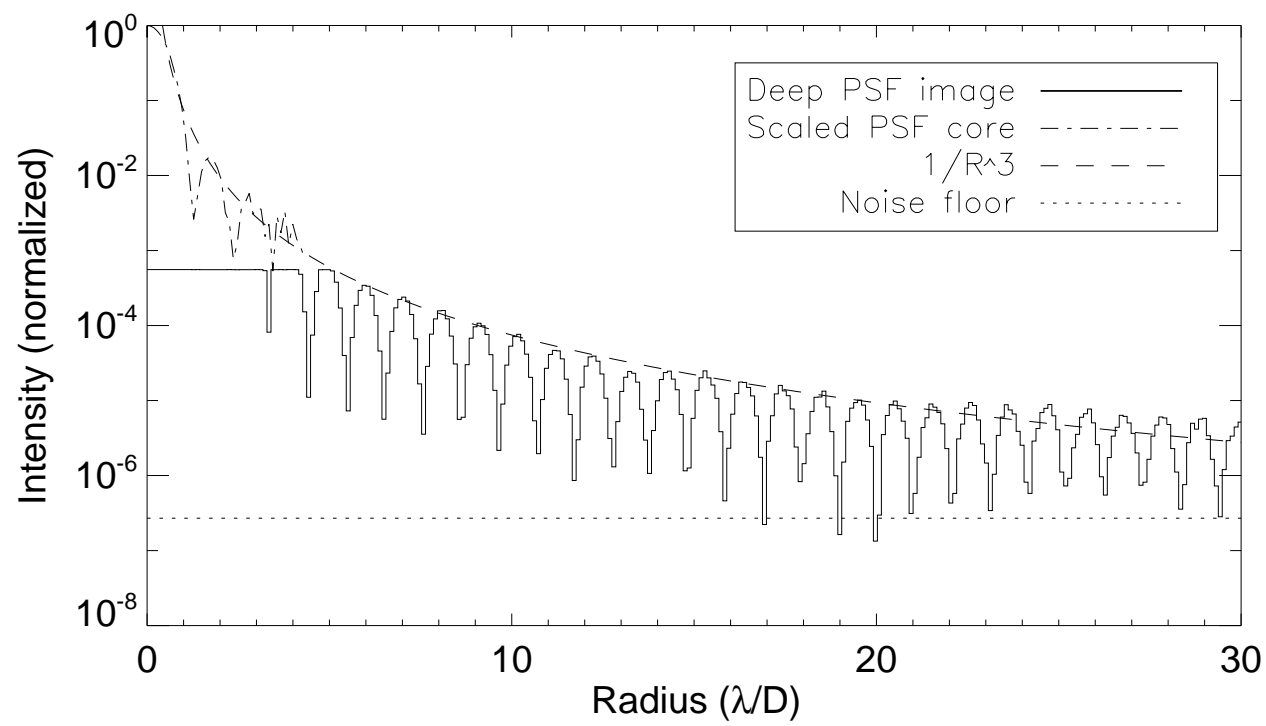

Figure 7: Slice through the PSF produced by the phase 1 testbed. The solid line shows the PSF at maximum intensity; the dash-dot line shows the PSF core, measured by attenuating the fiber source and rescaled. The dotted line shows the noise floor imposed by dark current and background fluctuations.

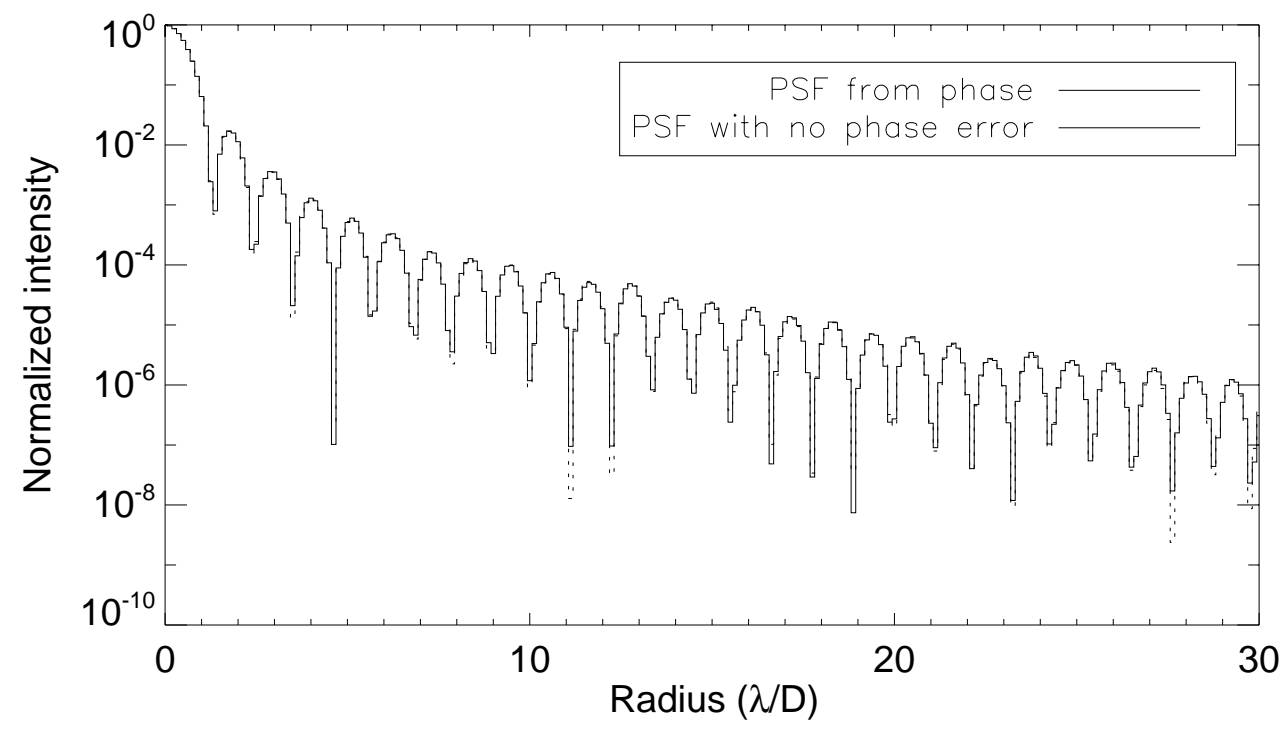

Figure 8: Simulated Airy patterns. The solid line shows the PSF generated using the measured phase and aperture. The dashed line shows the PSF for the aperture with no phase errors. 
The next phase of the testing will be to replace the flat mirror with a 1024-actuator MEMS DM, controlled by information from the PSDI sensor. Future phases will implement a coronagraph, a spatially-filtered Shack-Hartmann wavefront sensor, and ultimately a all-reflecting layout for use in white light rather than monochromatic laser light. Since MEMS are extremely thermally stable, we do not anticipate needing exquisite thermal control, and since our contrast goals are in the $10^{7}-10^{8}$ range and our beam size small, we should be able to operate in air rather than vacuum.

\section{CONCLUSIONS}

In the years since the concept was originally suggested by $\mathrm{Angel}^{8}$, technology has progressed to the point where construction of an "Extreme" AO system optimized for high-contrast imaging is now feasible. With a detailed understanding of the key issues in high-contrast $\mathrm{AO}$ we are using a science-based process to design such a system, which could be deployed on an $8-10 \mathrm{~m}$ telescope in 2007. XAOPI will achieve nearIR contrasts of $10^{7}-10^{8}$ for a large sample of target stars $\left(\mathrm{m}_{\mathrm{R}}<7\right)$, providing sensitivity to a large part of extrasolar planet phase space.

\section{ACKNOWLEDGEMENTS}

This research was performed under the auspices of the U.S. Department of Energy by the University of California, Lawrence Livermore National Laboratory under Contract W-7405-ENG-48, and also supported in part by the National Science Foundation Science and Technology Center for Adaptive Optics, managed by the University of California at Santa Cruz under cooperative agreement No. AST - 9876783. Support for this work was also provided by a grant from the Gordon and Betty Moore Foundation to the Regents of the University of California, Santa Cruz, on behalf of the Laboratory for Adaptive Optics. The content of the information does not necessarily reflect the position or the policy of the Gordon and Betty Moore Foundation, and no official endorsement should be inferred.

\section{REFERENCES}

1 Angel, J. R. P. 1994, Nature 368, 203

2 Macintosh, B., Olivier, S., Brase, J., Carrano, C., Carr, E., Gavel, D., Max, C.E., and Patience, J., 2002 proc. SPIE 4494, 60

3 Sivaramakrishnan, A., Lloyd, J., Hodge, P., and Macintosh, B. 2002 Ap.J. 581, L59

4 Sivaramakrishnan, A., Coresko, C., Makidon, R., Berkefeld, T., and Kuchner, M, 2001, Ap. J. 552,397

$5 \quad$ Varmderneo. R., Spergel, D., Kasdin, N.J., 2003 Ap.J. 590, 593

6. Burrows, A. et al. 1997, Ap.J., 491, 856

7. Perrin. M. D., Sivaramakrishnan, A., Makidon, R. B., Oppenheimer, B. R. \& Graham, J. R. 2003

Ap.J. in press

$8 \quad$ Angel, J. R. P. 1994, Nature 368, 203

9 Trauger, J., et al. 2003 Proc. SPIE 4854, 116

10 Roberts, L., and Neyman, C., 2002 PASP 114, 1260

11 Krulevitch PA, Bierden PA, Bifano TG, Carr E, Dimas CE, Dyson H, Helmbrecht MA, Kurczynski P, Muller RS, Olivier SS, Peter Y-A, Sadoulet B, Solgaard O, Yang E-H., 2003, Proc. SPIE 4985172

12 Poyneer, L., and Macintosh, B., 2003 Proc. SPIE 5169 in press

13 Poyneer, L, and Macintosh, B., 2003 J. Opt. Soc. Amer. A submitted

14 Marois, C., Doyon, R., Racine, R., \& Nadeau, D. 2000, PASP, 112, 91

15 Kuhn, J.R., Potter, D., \& Parise, B. 2001, ApJ, 553, 189L

16 Poyneer, L.A., Gavel, D., and Brase, J., 2002 J. Opt. Soc. Amer. A, 19, 2100

17 Rigaut, F., Veran, J., and Lai, O, 1998 Proc. SPIE 3353, 1038

18 Graham, J., Macintosh, B., and Kalas, P., 2003 PASP in prep 
19 Jaywardhana, R. and Greene, T.,2001, editors, "Young stars near earth", ASP conference series 244

20 Schneider, G., et al. 1999, ApJ, 513, L127

21 Sommargren, G.E., Phillion, D.W. ,Johnson, M.A., Nguyen, N.Q., Barty, A., Snell, F.J., Dillon, D.R., Bradsher, L.S., Phillion, D.W., Campbell, E.W., 2002, Proc. SPIE Vol. 4688, p. 316-328

22 Sommargren, G.E., in OSA Trends in Optics and Photonics Vol. 4, Extreme Ultraviolet Lithography, G.D. Kubiak and D.R. Kania, Editors (Optical Society of America, Washington, DC 1996), pp. 108-112 